\title{
Germanium/perovskite heterostructure for high-performance and broadband photodetector from visible to infrared telecommunication band
}

\author{
Wei Hu $\mathbb{1}^{1}$, Hui Cong ${ }^{2,3}$, Wei Huang ${ }^{1}$, Yu Huang ${ }^{1}$, Lijuan Chen ${ }^{1}$, Anlian Pan $^{4}$ and Chunlai Xue ${ }^{2,3}$
}

\begin{abstract}
A high-performance and broadband heterojunction photodetector has been successfully fabricated. The heterostructure device is based on a uniform and pinhole-free perovskite film constructed on top of a single-crystal germanium layer. The perovskite/germanium photodetector shows enhanced performance and a broad spectrum compared with the single-material-based device. The photon response properties are characterized in detail from the visible to near-infrared spectrum. At an optical fibre communication wavelength of $1550 \mathrm{~nm}$, the heterojunction device exhibits the highest responsivity of 1.4 A/W. The performance is promoted because of an antireflection perovskite coating, the thickness of which is optimized to $150 \mathrm{~nm}$ at the telecommunication band. At a visible light wavelength of $680 \mathrm{~nm}$, the device shows outstanding responsivity and detectivity of $228 \mathrm{~A} / \mathrm{W}$ and $1.6 \times 10^{10}$ Jones, respectively. These excellent properties arise from the photoconductive gain boost in the heterostructure device. The presented heterojunction photodetector provides a competitive approach for wide-spectrum photodetection from visible to optical communication areas. Based on the distinguished capacity of light detection and harvesting from the visible to near-infrared spectrum, the designed germanium/perovskite heterostructure configuration is believed to provide new building blocks for novel optoelectronic devices.
\end{abstract}

\section{Introduction}

A photodetector (PD) is an optoelectronic device widely used to convert light signals into electronic outputs. The photon response spectrum of a PD is critical for its detection application. This property is generally determined by the specific bandgap of a semiconducting active layer applied in a device ${ }^{1-3}$. A broadband photodetector, which can detect from visible (Vis) to infrared (IR) light, is particularly important in the commercial applications of imaging sensors, optical communication, environmental monitoring, and civil engineering ${ }^{4-6}$. However, a single semiconductor is hardly able to achieve a broader response spectrum as an active layer in a photodetector.

\footnotetext{
Correspondence: Chunlai Xue (clxue@semi.ac.cn)

${ }^{1}$ Key Laboratory for Micro-Nano Physics and Technology of Hunan Province, School of Physics and Electronics, Hunan University, Changsha, Hunan 410082, China

${ }^{2}$ State Key Laboratory on Integrated Optoelectronics, Institute of Semiconductors, Chinese Academy of Sciences, Beijing 100083, China Full list of author information is available at the end of the article.
}

For example, the inorganic semiconductor germanium (Ge) has been applied to construct a key component of photodetection in optical interconnection and optoelectronic integrated circuits (OEICs). It has unique optoelectronic properties at the IR telecommunication band and great process compatibility with complementary metal-oxide-semiconductor (CMOS) techniques ${ }^{7,8}$. Unfortunately, the results by far indicate that germanium has extremely poor response performance among the Vis light spectrum. The reasons for the shortcoming mainly lie in the short Vis light penetration length and low photogenerated carrier collection efficiency in germanium film (called the "dead region effect"). These limitations hinder it in the development of Vis-light communication, not to mention broadband absorption applications ${ }^{9-12}$.

To overcome the challenges mentioned above, much efforts have been made to construct heterojunction devices in recent decades. Many different kinds of materials, including layer materials such as graphene ${ }^{13}$ and

\section{(c) The Author(s) 2019}

\footnotetext{
(c) Open Access This article is licensed under a Creative Commons Attribution 4.0 International License, which permits use, sharing, adaptation, distribution and reproduction cc in any medium or format, as long as you give appropriate credit to the original author(s) and the source, provide a link to the Creative Commons license, and indicate if changes were made. The images or other third party material in this article are included in the article's Creative Commons license, unless indicated otherwise in a credit line to the material. If material is not included in the article's Creative Commons license and your intended use is not permitted by statutory regulation or exceeds the permitted use, you will need to obtain permission directly from the copyright holder. To view a copy of this license, visit http://creativecommons.org/licenses/by/4.0/.
} 
$\mathrm{MoS}_{2}{ }^{14}$, inorganic and organic semiconductors such as $\mathrm{PbS}^{15}, \mathrm{ZnO}^{16}, \mathrm{PDPP}^{17} \mathrm{~T}^{17}$ and $\mathrm{Si}^{18}$, have been explored together to capture more incident photons. For example, the graphene $/ \mathrm{Bi}_{2} \mathrm{Te}_{3}$ heterostructure photodetector shows a broadband response from 532 to $1550 \mathrm{~nm}^{13}$. However, it has low responsivity over the response spectrum, especially in the NIR spectrum $(0.22 \mathrm{~A} / \mathrm{W}$ at $1550 \mathrm{~nm}$ ) due to weak optical absorption of the layered graphene. The perovskite/ $\mathrm{MoS}_{2}$-based photodetector can detect visible light only due to the bandgap limitation of the two layers, and shows a peak responsivity of $68 \mathrm{~A} / \mathrm{W}$ at a wavelength of $514 \mathrm{~nm}^{14}$. Moreover, a high temperature over hundreds of degrees is typically used for the deposition of $\mathrm{ZnO}, \mathrm{TiO}_{2}$ and Si film, which would cause critical damages to the underlying layers or substrates. Based on the research works conducted so $\mathrm{far}^{13-23}$, the limited response spectrum, low performance, high working voltage and incompatibility of the procedure at low temperatures have become major concerns for the heterojunction photodetector construction. These disadvantages also strongly hamper their widespread commercialization applications.

Recently, a series of solution-processed organic-inorganic hybrid perovskite has attracted extensive attention in the research area of optoelectronic devices. Methylammonium lead triiodide $\left(\mathrm{CH}_{3} \mathrm{NH}_{3} \mathrm{PbI}_{3}\right)$ is the most representative one among these materials. The $\mathrm{CH}_{3} \mathrm{NH}_{3} \mathrm{PbI}_{3}$ perovskite thin film can be easily synthesized, which has excellent advantages such as a direct bandgap $^{24}$, long charge carrier diffusion length ${ }^{25}$, low recombination rate and high absorption coefficient in the Vis light range ${ }^{26}$. Its application in the areas of optical amplification $^{27}$, nonlinear optical areas ${ }^{28}$, and light- emitting diodes ${ }^{29}$ has been studied. In the last two decades, the $\mathrm{CH}_{3} \mathrm{NH}_{3} \mathrm{PbI}_{3}$ thin film has been mostly explored as light harvester of a solar cell ${ }^{30,31}$, which has an energyconversion efficiency of over $25 \%^{32}$. The perovskite thin film has also been explored as an active layer in photodetectors with vertical (photovoltaic type) and lateral (metal-semiconductor-metal type) device architectures ${ }^{33-}$ ${ }^{43}$. The devices exhibited good photo response properties under Vis light illumination. However, they cannot absorb the photons in the IR spectrum. The reported photodetectors show a cut-off wavelength of $780 \mathrm{~nm}$ due to the bandgap limitation of the perovskite absorber ${ }^{37-40}$. This means that the $\mathrm{CH}_{3} \mathrm{NH}_{3} \mathrm{PbI}_{3}$ perovskite features strong absorption in the Vis light spectrum and high transparency in the near-IR spectrum. Therefore, the perovskite material is an appropriate candidate, being constructed with germanium, which is a heterostructure photodetector aiming to match the Vis-to-IR broad photo response requirement.

\section{Results}

A perovskite/germanium heterojunction photodetector with excellent photo-response properties has been successfully fabricated. As shown in Fig. 1, the fabrication process of a germanium/perovskite heterojunction photodetector is schematically exhibited. First, a germanium layer with a thickness of $300 \mathrm{~nm}$ is grown by a solidsource molecular beam epitaxy (MBE) technique. The germanium on insulator (GOI) samples are fabricated by a wafer bonding process ${ }^{44,45}$. Second, interdigital gold electrodes are formed by using a thermal vapor deposition method. The channel length and width are defined by a shadow mask. Third, a $\mathrm{PbI}_{2}$ layer is deposited on top of

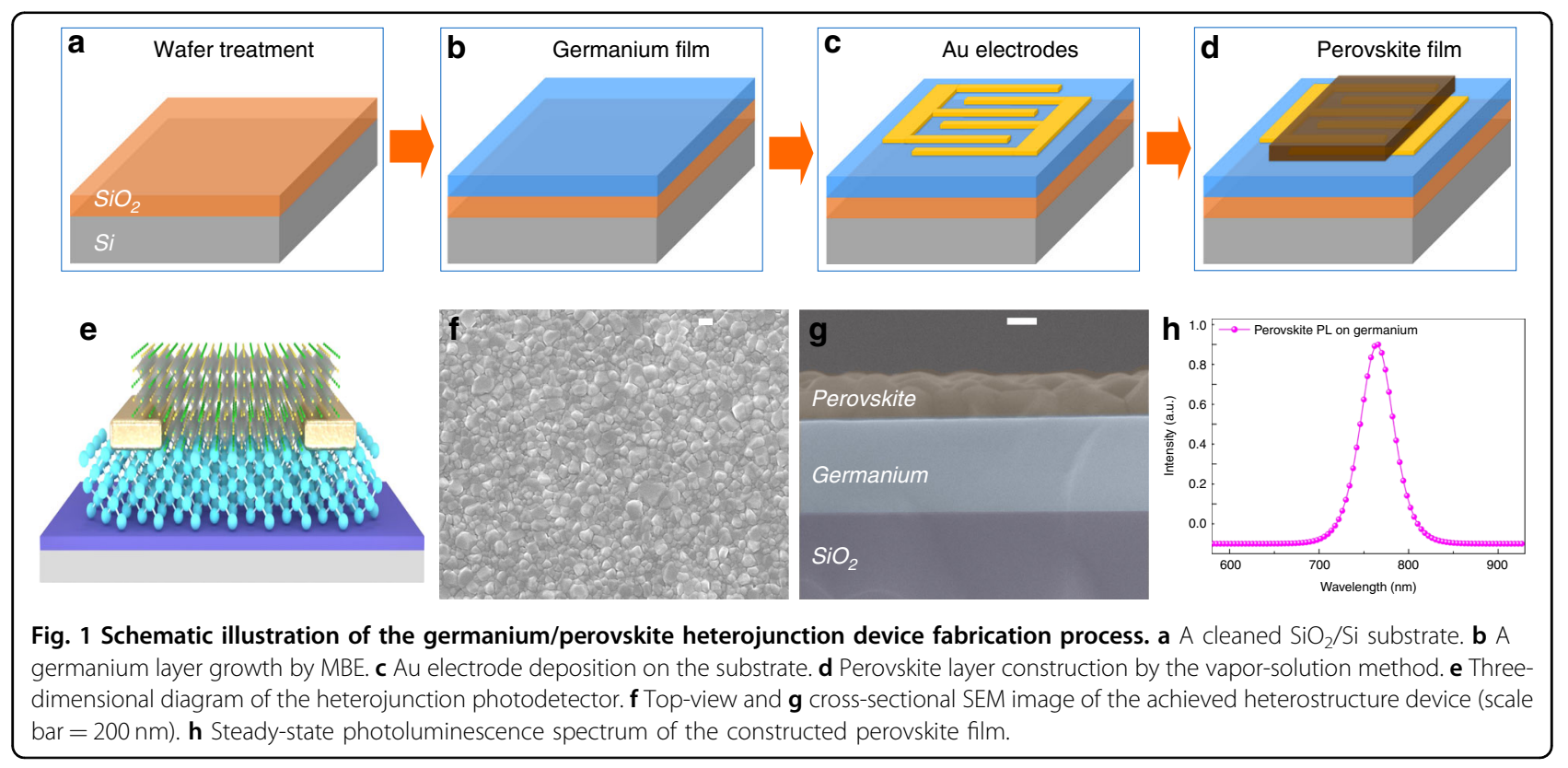


the sample, the thickness of which is monitored precisely by a quartz crystal oscillation. Then, a drop of $\mathrm{CH}_{3} \mathrm{NH}_{3} \mathrm{I}$ solution (in isopropanol) is spin-coated onto the asconstructed $\mathrm{PbI}_{2}$ film. Finally, the heterojunction photodetector is constructed via an annealing process performed for $30 \mathrm{~min}$ at $100{ }^{\circ} \mathrm{C}$. More details can be found in the experiment section and our previous works ${ }^{43}$.

Three-dimensional diagram of a perovskite/germanium heterojunction photodetector is shown in Fig. 1e. A topview scanning electron microscopy (SEM) image of the perovskite layer is illustrated in Fig. 1f. A cross-sectional SEM image of the device is presented in Fig. 1g. These images present a compact and pinhole-free perovskite film fully covering on the germanium layer. Figure $1 \mathrm{~h}$ shows the steady-state photoluminescence (PL) spectrum of the constructed perovskite layer. The peak wavelength is located at $765 \mathrm{~nm}$, which is consistent with previous results $^{27-31}$. For comparison, devices based on pristine perovskite and germanium active layers have been fabricated. The optical and electronic properties of these obtained photodetectors were further performed under the same conditions as those of the heterojunction devices. All these measurements were characterized in air without being encapsulated at room temperature.

In a germanium-based IR photodetector, a considerable portion of incident IR light is reflected from the germanium surface. The germanium active layer has a relatively high refractive index $\left(n_{G=4.0)}\right.$ and a low absorption coefficient $^{44,45}$. The light off the device suppresses photocurrent formation, which is undesirable in photonabsorption-based devices. Use of an antireflection (AR) coating is an efficient approach to reducing light reflection and maximize transmission into the active layer ${ }^{46-48}$. As we know, the $\mathrm{CH}_{3} \mathrm{NH}_{3} \mathrm{PbI}_{3}$ perovskite layer is transparent at the telecommunication band due to its bandgap limitation of $1.5 \mathrm{eV}$. Meanwhile, it has a relatively lower refractive index $\left(n_{p}\right)$ of 2.3 compared with that of germanium $^{49,50}$. These advantages suggest that the perovskite material is an appropriate AR coating for germanium-based high-performance photodetectors. To take advantage of both materials to overcome the challenges discussed above, we introduce a germanium/perovskite heterojunction based on the following considerations. First, the perovskite and germanium films are used as absorbers for the effective absorption of Vis and IR light, respectively. Second, the top perovskite layer is applied as an AR coating with regard to the IR spectrum due to its lower refractive index and wider bandgap than those of germanium. At a wavelength of $1550 \mathrm{~nm}$, the thickness of the perovskite AR coating is optimized to achieve the lowest reflection in the photodetector. Third, type-I energy band alignment forms in the germanium/ perovskite heterojunction. It can be noted that there is a large band offset between the conduction and valence bands of the two semiconducting materials. This offset easily produces a charge carrier transport from the perovskite into the germanium. The ultra-high carrier mobility in germanium results in a photoconductive gain boost in the heterojunction photodetector. Above all, the germanium/perovskite hetero-structure configuration not only benefits the broadband photodetector but also provides a possible method of developing novel optoelectronic applications. The mechanism of the germanium/ perovskite heterojunction photodetector is described in detail below.

As shown in Fig. 2a, the incident IR light is partly reflected off the device because of the refractive difference between the semiconductor layer and air. Based on the fundamental principles of optics ${ }^{51}$, the reflectivity portion is determined by the perovskite AR coating thickness $(l)$ and the incident wavelength $(\lambda)$. To clarify the function of the perovskite AR coating, a calculation is first carried out to evaluate the relationship between the reflectivity and perovskite film thickness. As shown in Fig. 2c, the basic reflectance off surface of the pristine germanium layer is $\sim 36 \%$ at a telecommunication wavelength of $1550 \mathrm{~nm}$. The value becomes significantly lower when a perovskite layer is coated onto the germanium as the AR coating. When the perovskite film thickens to approximately $150 \mathrm{~nm}$, the lowest reflectance of $7 \%$ is obtained. Then, the value rises slightly and reaches the highest value (35\%) as the AR film thickness exceeds $300 \mathrm{~nm}$. The images of the optical field distribution in the bilayer device are also simulated and presented. The full 3D electrodynamics finite element method (FEM) simulations are performed using the COMSOL Multiphysics software. Figure 2b and Fig. S1 show the simulation results for the photodetector with different AR coating thicknesses $(0,100,120,150$, 180 , and $300 \mathrm{~nm}$ ) at a wavelength of $1550 \mathrm{~nm}$. It can be seen that a $150 \mathrm{~nm}$ AR coating exhibits the lowest IR photon loss. The theoretical results predict that the lowest reflectance can be achieved with an optimized perovskite AR thickness about $150 \mathrm{~nm}$. Meanwhile, the enhanced transmit effect in the IR spectrum is observed, as shown in Fig. S2. This effect indicates that a perovskite AR coating with an optimized thickness at a wavelength of $1550 \mathrm{~nm}$ can effectively reduce the photon loss in the range of IR spectrum. Based on the analysis above, a batch of heterojunction photodetectors with different of perovskite AR coating thicknesses are constructed and characterized. The perovskite thickness can be precisely controlled in the first step during the $\mathrm{PbI}_{2}$ layer fabrication process. The cross-sectional SEM figures of the devices are shown in Fig. $1 \mathrm{~g}$ and Fig. S3 for different perovskite thicknesses. The photocurrents $\left(I_{\mathrm{ph}}\right)$, defined as the current difference between illumination and darkness, are presented in Fig. $2 \mathrm{~d}$ under an incident wavelength of $1550 \mathrm{~nm}$. Compared with the pristine germanium device, $I_{\mathrm{ph}}$ increases 


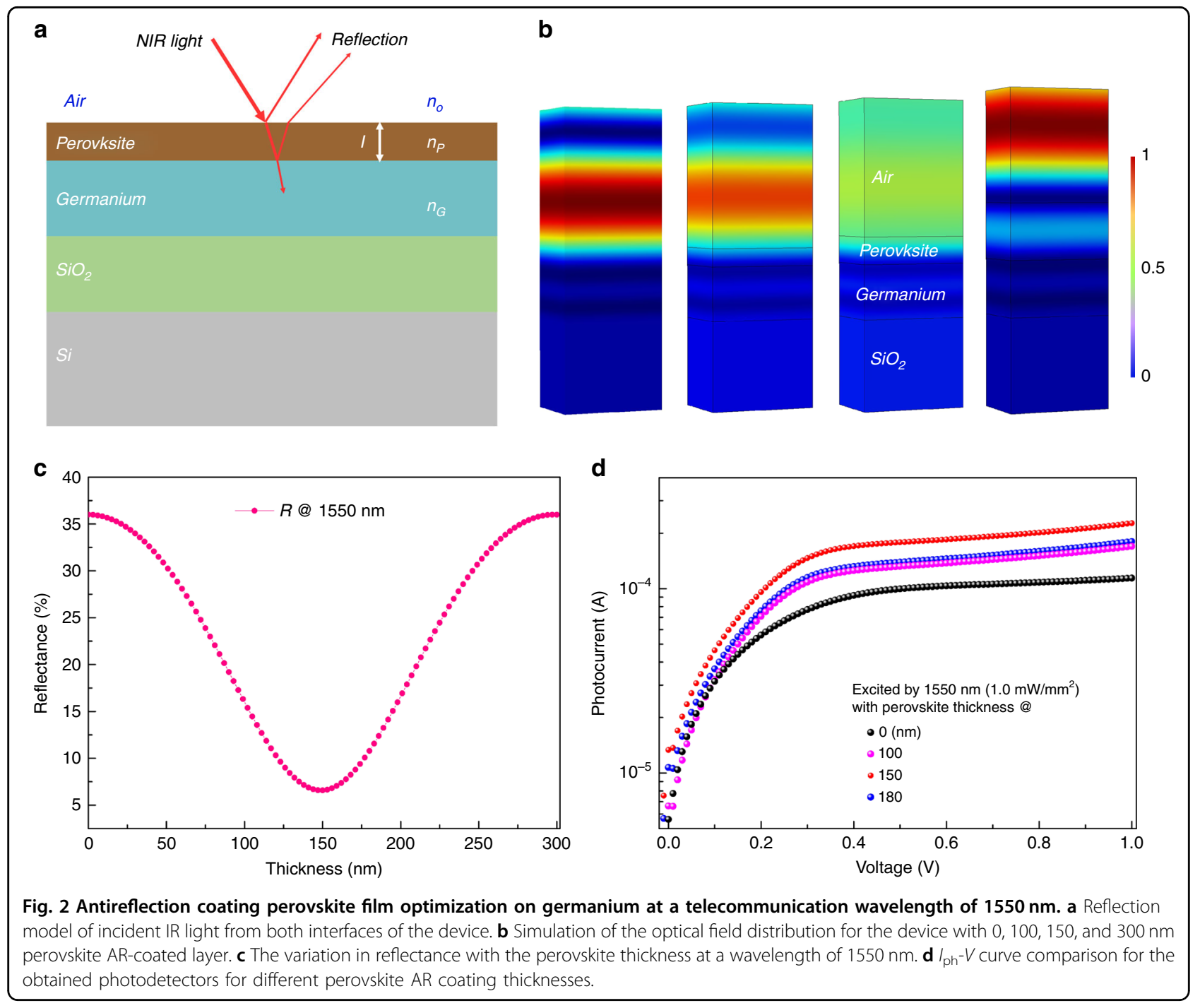

obviously in the perovskite AR-coated device. The highest $I_{p h}$ value is obtained in a heterojunction device when the perovskite AR coating thickness is approximately $150 \mathrm{~nm}$. The experimental results also match the theoretical predication discussed above. Figure S4 presents the $I_{\mathrm{ph}^{-}}-V$ comparison for the pristine germanium and AR coating optimized device. The higher $I_{\mathrm{ph}}$ of the perovskite/germanium heterojunction device is obvious.

Meanwhile, the photodetector performance is characterized at an optical communication wavelength of $1550 \mathrm{~nm}$. Figure 3a shows the illumination-powerdependent $I_{\mathrm{ph}}-V$ curves of the device. This figure indicates that the $I_{p h}$ increase with the applied bias and incident power. Figure $3 \mathrm{~b}$ (solid circle) exhibits the dependence of $I_{p h}$ on the illumination power at a bias of $1 \mathrm{~V}$. It shows a good linear relationship as the power density increases from 0.08 to $3.24 \mathrm{~mW} \mathrm{~cm}^{-2}$, which suggests that our heterojunction $\mathrm{PD}$ is capable of detecting incident light power over a wide range. The important figure of merits, the photodetector spectral responsivity $(R)$ and the detectivity $\left(D^{\prime \prime}\right)$ have also been characterized. $R=I_{\mathrm{ph}} / P_{\mathrm{In}}$ is defined as the ratio of the device photocurrent to the incident light intensity, in which $P_{I n}$ is the incident optical power. Figure $3 \mathrm{~b}$ shows the varying trend of $R$ (star) with incident power under a bias of $1 \mathrm{~V}$. A maximum value of $R=1.4 \mathrm{~A} \mathrm{~W}^{-1}$ at $1550 \mathrm{~nm}$ is achieved for the heterojunction photodetector. As far as we know, this is the highest $R$ among the reported IR photodetectors to date at a low working voltage of $1 \mathrm{~V}^{7-12}$. The responsivity drops significantly as the incident light intensity increases. This result suggests that the photocurrent flowing through the device does not proportionately increase as the incident light intensity increases. The major potential reason is the limited absorption coefficient of the active layer. As the proportion of illumination photons increases, the absorption gradually saturates in the germanium active layer. Then, the photogenerated carrier and photocurrent do not 

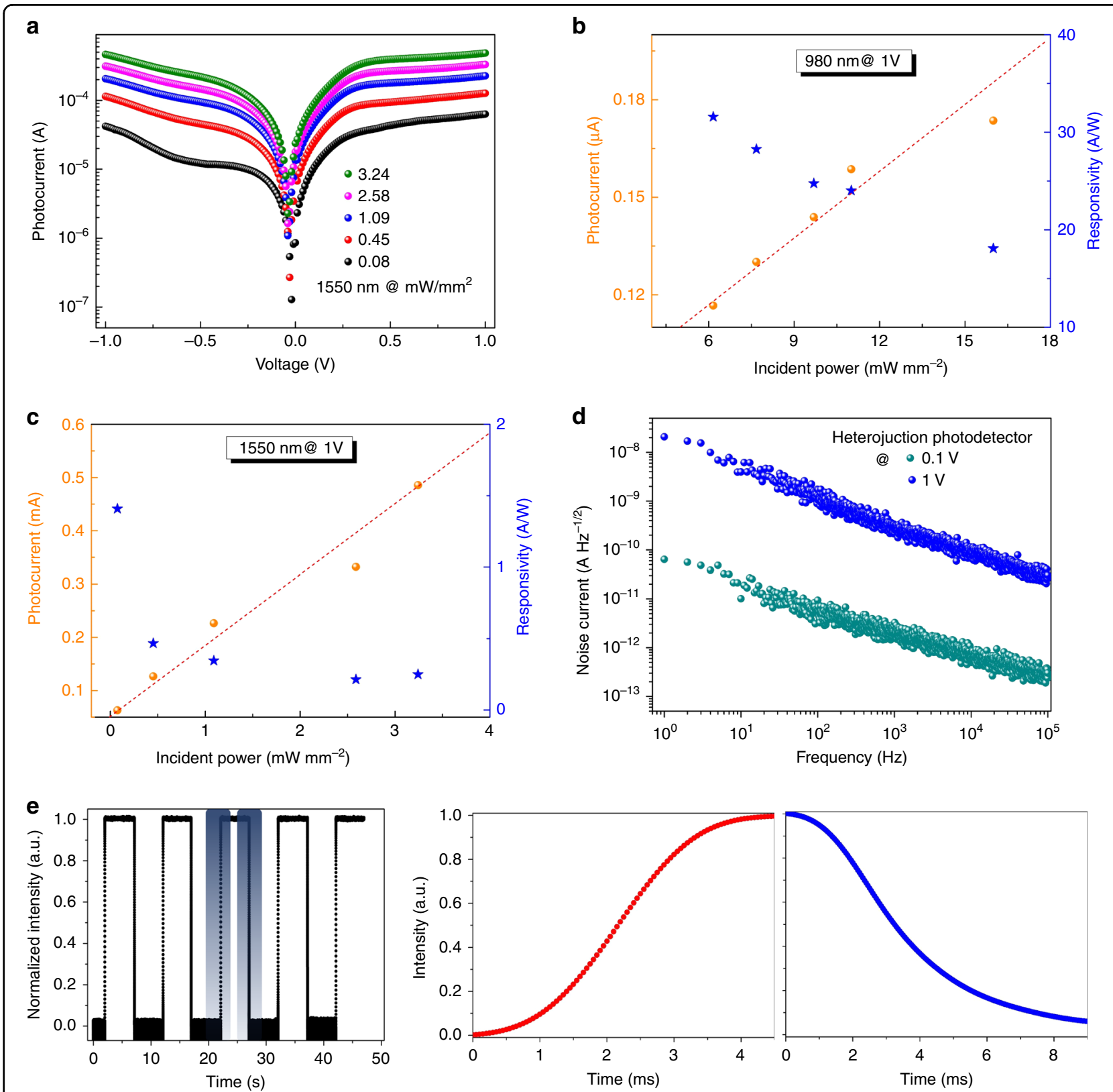

Fig. 3 The performance of the optimized heterojunction photodetector in the infrared spectrum. a Device $I-V$ curves and $\mathbf{b} I_{p h}$ and $R$ values under an illumination wavelength of $1550 \mathrm{~nm}$. c Noise currents of the heterojunction device, varying with the frequency $(0.1$ and $1 \mathrm{~V})$. d $/$ ph and $R$ of the device under an incident wavelength of $980 \mathrm{~nm}$. e Device response speed under an incident wavelength of $1550 \mathrm{~nm}$. The zoomed-in views of the rise and decay versus time correspond to start times of 22.110 and $27.029 \mathrm{~s}$, respectively.

increase accordingly, which results in a decrease in the $R$ values. The photodetector performance is presented in Fig. 3d at an incident wavelength of $980 \mathrm{~nm}$. The highest $R$ value of $32 \mathrm{~A} \mathrm{~W}^{-1}$ is achieved at a bias of $1 \mathrm{~V}$. Another important parameter, $D^{*}$, is used to quantitatively evaluate the capability of a detector in weak light detection. It is determined by the responsivity and noise of a photodetector, $D^{*}=(A \Delta f)^{\frac{1}{2}} R / i_{n}$, in which $A$ is the device effective area and $\Delta f$ and $i_{n}$ are the electrical bandwidth and noise current of the device, respectively. Figure $3 \mathrm{c}$ shows the noise currents of a heterojunction device at various frequencies. The results for the pristine germanium device are shown in Figure S5. The curves indicate that noise currents decrease as the frequency increases and reach a higher level under a larger voltage due to a higher dark current. At a wavelength of $1550 \mathrm{~nm}$, the $D^{*}$ of a germanium/perovskite photodetector is estimated to be $1 \times 10^{8}$ Jones $\left(\mathrm{cm} \mathrm{Hz}^{1 / 2} \mathrm{~W}^{-1}\right)$ at $0.1 \mathrm{~V}$, which is 

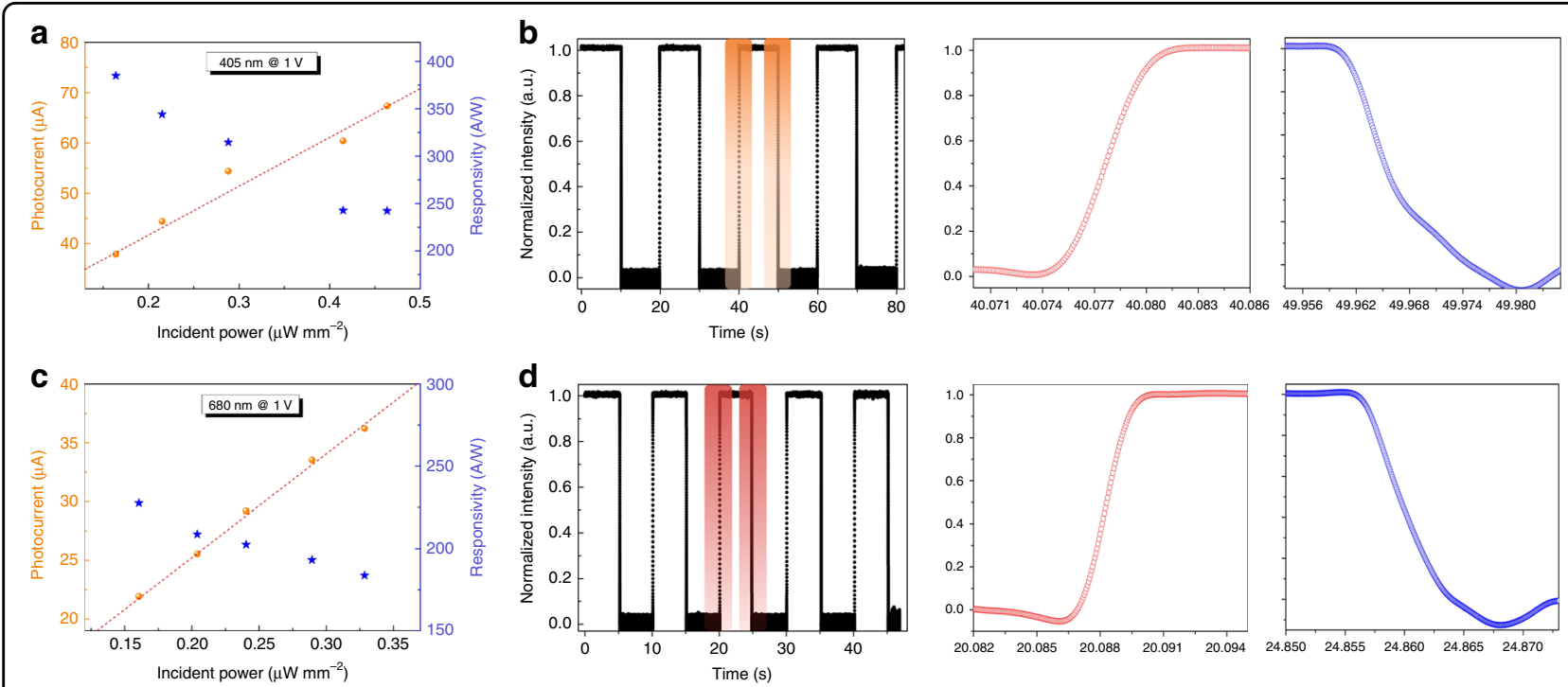

Fig. 4 Performance of the heterojunction photodetector in the visible light spectrum. $I_{p h}$ and $R$ of the device under illumination wavelengths of $\mathbf{a} 405 \mathrm{~nm}$ and $\mathbf{c} 680 \mathrm{~nm}$. Response speed of the heterojunction photodetector. The rise and decay times of the device under an incident wavelength of $\mathbf{b} 405$ and $\mathbf{d} 680 \mathrm{~nm}$.

better than the reported results for the telecommunication band. The higher values of $R$ and $D^{*}$ suggest that our heterojunction photodetector has enhanced performance compared with that of the pristine germanium device. We ascribe the promotion mainly to the introduction of the perovskite AR coating. The perovskite thickness is optimized to reduce the IR photon loss efficiently due to reflection. As more IR photons are transmitted and trapped in the germanium film, more photon-induced charge carriers are generated in the active layer. Consequently, the constructed heterojunction photodetector exhibits higher photocurrent and better performance than those of the pristine germanium detector. Device response speed experiments are also carried out. Figure 3e shows the time-resolved on-off switching behaviors of the photodetector being investigated at an IR wavelength of $1550 \mathrm{~nm}$. The rise and decay time of the heterojunction photodetector are measured to be 2.1 and $5.7 \mathrm{~ms}$ at room temperature, respectively, which show higher speeds than those of pristine germanium device ${ }^{7-10}$.

The heterojunction photodetector performance in the Vis light region has also been characterized. The perovskite layer is chosen to have an optimized thickness of $150 \mathrm{~nm}$, which is approximately two times thinner than the reported devices, including perovskite solar cells and photodetectors $^{21-26}$. Figure 4a, c summarizes the $I_{p h}$ and $R$ values of the device under two typical Vis wavelengths, namely, 405 and $680 \mathrm{~nm}$. Figure $S 6$ shows the $I-V$ curve comparison between the pristine germanium and the heterojunction photodetectors. The photocurrents of the heterojunction device are noted to be higher than those of the pristine germanium device. The $I-V$ performance of a heterojunction device at different illumination powers is presented in Fig. S7 for varying wavelength in the Vis spectrum. The figures indicate that $I_{p h}$ increases dramatically with the incident power density. The typical values of $R$ and $D^{*}$ under Vis light illumination are obtained for the heterojunction device. For example, a high $R$ of $228 \mathrm{~A}$ $\mathrm{W}^{-1}(1 \mathrm{~V})$ at an illumination wavelength of $680 \mathrm{~nm}$ is achieved. $D^{*}$ is estimated to be $1.6 \times 10^{10}$ Jones at a $0.1 \mathrm{~V}$ bias. These parameters are comparable with the those of the pristine perovskite photodetectors, the active layer thickness of which is approximately two times that of this device ${ }^{21-29}$. Figure $4 \mathrm{~b}, \mathrm{~d}$ shows the on-off switching behaviors of the heterojunction device. Under incident light of $680 \mathrm{~nm}(405 \mathrm{~nm})$, the device rising and decay times are measured to be 1.8 and $5.1 \mathrm{~ms}$ ( 4.1 and $9.2 \mathrm{~ms}$ ), respectively. The constructed photodetector exhibits higher performance and faster response in the Vis spectrum than those of the pristine device. We ascribe these promotions to bilayer structures based on perovskite and germanium layers in the detector. The device physics based on the energy-band model and optical techniques are studied comprehensively below. Figure 5a shows the energy band of isolated germanium and perovskite materials. The conduction and valence band edges for intrinsic perovskite (germanium) are 3.87 (4.13) eV and $5.45(4.80) \mathrm{eV}^{40-44}$, respectively. There is a relatively high energy band difference between the two semiconductor materials $(0.26 \mathrm{eV}$ for the conduction band and $0.65 \mathrm{eV}$ for the valence band). The Fermi energy difference is $0.18 \mathrm{eV}$ based on the obtained ultraviolet photoelectron 

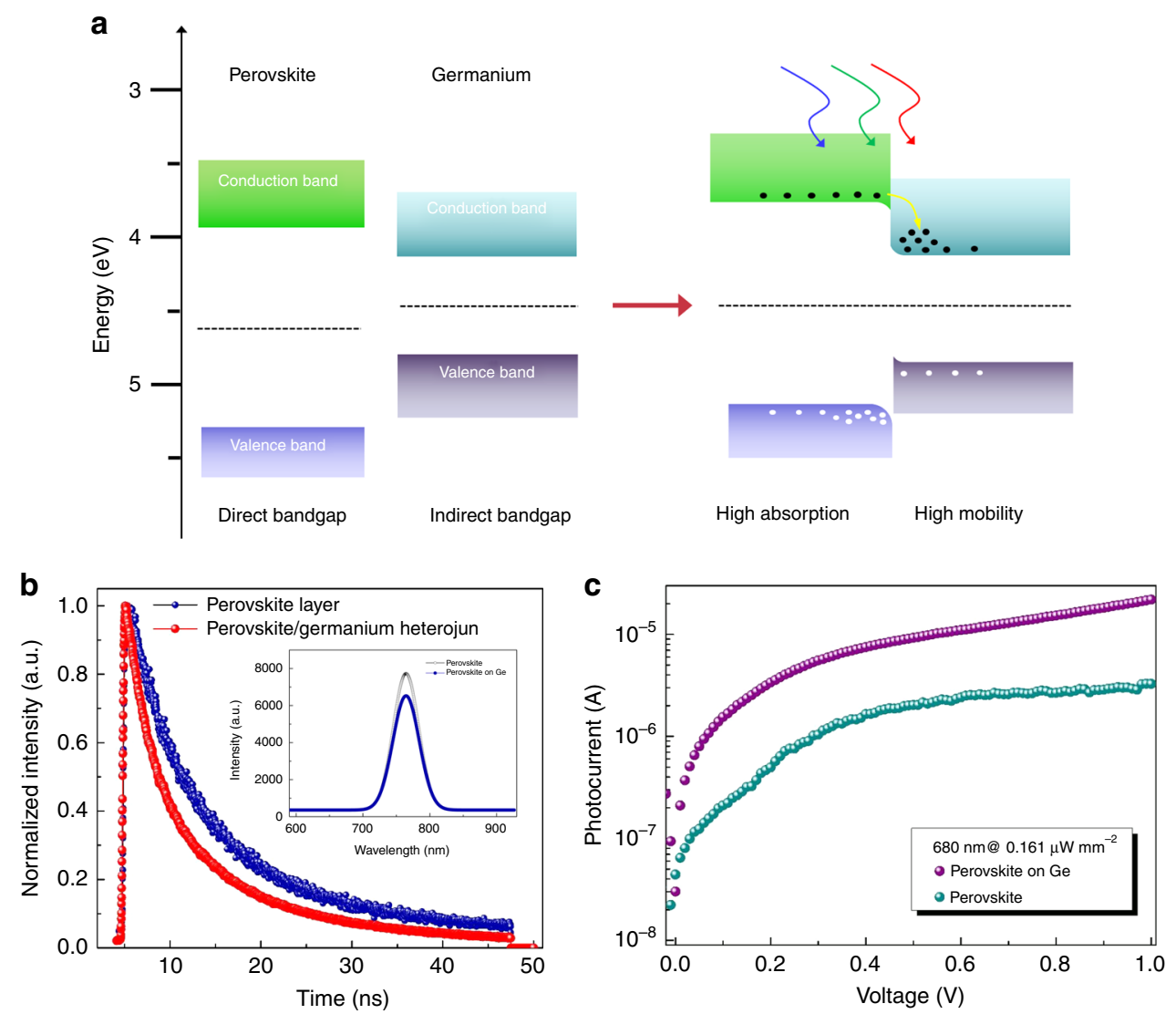

Fig. 5 The germanium/perovskite heterojunction photodetector energy band model and optical characterization. a Energy-band diagrams for two isolated semiconductors and heterojunction device (type I energy band). b Time-resolved PL decay and intensity (inset) comparison for the pristine and hetero-structure perovskite. $\mathbf{c} I_{\text {ph }}-V$ curves for pristine perovskite and heterojunction photodetector.

spectroscopy (UPS, Fig. S8). At thermal equilibrium, energy band bending occurs at the heterojunction interface due to the requirement of the Fermi level coincident on both sides $^{52-55}$. Then, the conduction band energy difference at the interface is approximately $0.44 \mathrm{eV}$, arising from band bending, as shown in Fig. 5a. Driven by the large band offset, the photogenerated free electrons in the perovskite layer are transferred easily into the germanium layer. However, the holes in the valence band of the perovskite layer are confined in the valence band due to barrier formation as the band bends downwards. Optical and photoelectronic methods have been carried out to evaluate the effect of interface properties on the device performance. Figure $5 \mathrm{~b}$ shows the time-resolved PL and decay transient curves for pristine perovskite and on the germanium layer. The experiments are all performed under the same situation. The inset of Fig. $5 \mathrm{~b}$ shows the PL spectrum with a peak wavelength of $765 \mathrm{~nm}$, which corresponds to the band-edge emission of the $\mathrm{CH}_{3} \mathrm{NH}_{3} \mathrm{PbI}_{3}$ layer. A slightly lower intensity can be observed for perovskite constructed on germanium. This observation suggests that the density of the photon- generated carrier becomes lower in the perovskite layer constructed on the germanium layer. Based on the curves fitted by the bi-exponential decay function, the carrier lifetime is obtained as shown in Table S1. The fast decay component is associated with trap-assisted recombination, and the slow decay part is ascribed to radiative recombination. In terms of the perovskite on germanium, the obtained decay times are 11.5 and $2.87 \mathrm{~ns}$ (fast and slow lifetimes, respectively), which are obviously shorter than the values of 19.0 and $6.1 \mathrm{~ns}$ for the pristine perovskite on glass. The PL decay curves suggest that the photogenerated carriers have a marginally faster recombination rate in the perovskite layer of the heterojunction device than that in pristine film. This observation indicates that a number of photogenerated carriers will be delocalized from the perovskite absorption layer. The lower charge carrier lifetime also explains the faster response speed of the heterojunction device. There are two potential reasons for this lower PL intensity and shorter charge carrier lifetime. The first one is that a number of charge carriers are localized by trap states at the interface. The second is that part of the charge carriers 
will be transported from the perovskite to the germanium layer due to the existence of a large band offset. Benefitting from electron transportation from perovskite to germanium, an enhanced photoconductive gain has also been estimated. The gain is defined as ${ }^{1,2} G=I_{\mathrm{ph}} / I_{\mathrm{PI}}=\tau / t_{p}$ $=\tau \xi\left(\mu_{n}+\mu_{p}\right) / L$, where $I_{P I}$ is the primary photocurrent, $\tau$ is the carrier lifetime, $t_{p}$ is the carrier transit time across the electrodes, $\xi$ is the applied electric field, $L$ is the channel width, and $\mu_{n}$ and $\mu_{p}$ are the electron and hole mobility, respectively. Under Vis light excitation, some of the photogenerated electrons are transferred from the perovskite layer to the germanium layer. Compared with perovskite, the ultra-high electron mobility $\left(\approx 3800 \mathrm{~cm}^{2}\right.$ $\left.\mathrm{V}^{-1} \mathrm{~s}^{-1}\right)$ and long carrier lifetime $(\approx 200 \mu \mathrm{s})$ in the germanium layer result in an enhanced gain of $\sim 10^{4}$ in our heterojunction photodetector. This is approximately two orders higher than that in the pristine perovskite photodetector. This result indicates a photoconductive gain boost obtained in the heterojunction device. It proves the superior photocurrent (Fig. 5c) and device performance that have been achieved in the Vis light region even with a thinner perovskite layer.

\section{Discussion}

To compare the performance of our work and those reported, the main parameters of the heterostructure broadband photodetectors are summarized in Table 1. Notably, the achieved device in this work shows significant performance promotion compared with previously reported works on the Vis and IR spectra. As discussed above, the reasons and mechanisms behind device performance enhancement are attributed to the perovskite/germanium heterostructure.

In this study, we design and construct a heterostructure photodetector successfully by combining inorganic semiconductor germanium with hybrid inorganic-organic perovskite $\mathrm{CH}_{3} \mathrm{NH}_{3} \mathrm{PbI}_{3}$. A vapor-solution process provides a uniform and pinhole-free perovskite film on a germanium layer. The constructed heterojunction photodetector shows broader bandwidth and enhanced performance compared with those of the single-materialbased device. The detection properties of the heterojunction photodetector are characterized at a Vis light wavelength of $680 \mathrm{~nm}$ and an optical communication band of $1550 \mathrm{~nm}$. Under Vis light illumination, the free electrons photogenerated in the perovskite are partly transferred to the germanium, resulting in a photoconductive gain boost. The device shows outstanding responsivity and detectivity of $228 \mathrm{~A} / \mathrm{W}$ and $1.6 \times 10^{10}$ Jones at a wavelength of $680 \mathrm{~nm}$, respectively. When the perovskite AR coating thickness is optimized, the heterojunction device possesses the highest responsivity of $1.4 \mathrm{~A} / \mathrm{W}$ at an optical fiber communication band of $1550 \mathrm{~nm}$. The germanium/perovskite heterostructure has a broadband detection range from the ultraviolet to the Vis and then to the IR spectrum. Its high performance shows great potential application in wide-spectrum photodetection, ultraviolet-Vis or optical communication, tandem solar cells, and next-generation optoelectronic devices.

\section{Materials and methods \\ Fabrication procedure}

The $\mathrm{Si}$ coated with $\mathrm{SiO}_{2}$ substrates is cleaned by the traditional method. First, a $300 \mathrm{~nm}$ Ge is deposited by means of epitaxy onto the cleaned Si substrate using a solid-source MBE system, which is covered by a $350 \mathrm{~nm}$ thick $\mathrm{SiO}_{2}$ film deposited by using a plasma enhanced chemical vapor deposition (PECVD) system. This wafer is directly bonded to a handle $\mathrm{Si}$ substrate using the benzocyclobutene $(\mathrm{BCB})$ wafer bonding technique, followed by thermal treatment at $260^{\circ} \mathrm{C}$ under vacuum for $6 \sim 8 \mathrm{~h}$. Then, the initial Si substrate on the Ge film is completely removed by the inductively coupled plasma (ICP) dry etching and tetramethylammonium hydroxide (TMAH) wet etching techniques. Then, gold interdigital electrodes with an electrode width $(W)$ and inter-electrode space length $(L)$ of 2 and $0.05 \mathrm{~mm}$, respectively, are formed by thermal evaporation. The active area of the heterojunction photodetector is $0.6 \mathrm{~mm}^{2}$. A highly crystalline and compact perovskite $\mathrm{CH}_{3} \mathrm{NH}_{3} \mathrm{PbI}_{3}$ thin film is constructed by a two-step method on the germanium layer. A high-purity and homogeneous $\mathrm{PbI}_{2}$ film was first prepared by using a thermally physical vapor phase growth at a pressure of $10^{-4} \mathrm{~Pa}$. The deposition rate and film thickness are monitored by a quartz crystal oscillator. The samples are kept at room temperature during the deposition process. An isopropanol solution of $\mathrm{CH}_{3} \mathrm{NH}_{3} \mathrm{I}(10 \mathrm{mg} / \mathrm{ml})$ is then spin-coated onto the high-quality $\mathrm{PbI}_{2}$ films at $3000 \mathrm{rpm}$ for $30 \mathrm{~s}$. Immediately, the samples are moved to a hot plate for annealing at $100{ }^{\circ} \mathrm{C}$ for $30 \mathrm{~min}$ in an ambient environment. The $\mathrm{PbI}_{2}$ and $\mathrm{CH}_{3} \mathrm{NH}_{3} \mathrm{I}$ powders are purchased from Xi'an Polymer Light Technology Corporation and used without further purification.

\section{Material and device characterizations}

The morphology is characterized by a scanning electron microscope (SEM, Hitachi S-4800, Japan). The performance measurements are all performed in a clean room at a constant room temperature of $23^{\circ} \mathrm{C}$. The currentvoltage characteristics are determined on a probe station, and the data are recorded by a semiconductor parameter analyser (Keithley 4200) in the atmosphere. A xenon lamp (PL-SPS1000, Perfect Light Co. Ltd., China) with a monochromatic light source is used as the Vis light source. Two semiconductor lasers (980 and $1550 \mathrm{~nm}$ ) are used at the IR light source. The incident light power is calibrated before the measurements by a standard silicon 
Table 1 Performance comparison of heterostructure broadband photodetectors.

\begin{tabular}{|c|c|c|c|c|c|}
\hline Device & Wavelength (nm) & $R(\mathrm{~A} / \mathrm{W})$ & $D^{*}$ (Jones) & On/off time (ms) & Refs. \\
\hline \multirow[t]{3}{*}{ Graphene/Bi $\mathrm{Te}_{3}$} & 532 & 35 & - & $8.7 / 14.8$ & 13 \\
\hline & 980 & 8.5 & & & \\
\hline & 1550 & 0.22 & & & \\
\hline Perovskite/MoS 2 & 514 & 68.11 & - & $0.205 / 0.206$ & 14 \\
\hline \multirow[t]{3}{*}{$\mathrm{ZnO} / \mathrm{PbS}$} & 350 & $51 \times 10^{-6}$ & $3.4 \times 10^{8}$ & $<500$ & 15 \\
\hline & 500 & $7.2 \times 10^{-6}$ & $4.9 \times 10^{7}$ & & \\
\hline & 900 & $11 \times 10^{-6}$ & $7.2 \times 10^{7}$ & & \\
\hline \multirow[t]{2}{*}{ Perovskite/ZnO } & White light & 28 & $1.1 \times 10^{12}$ & $0.4 / 0.5 \times 10^{3}$ & 16 \\
\hline & 1357 & 0.22 & $9.3 \times 10^{9}$ & $0.3 / 0.8 \times 10^{3}$ & \\
\hline \multirow[t]{3}{*}{ Perovskite/PDPP3T } & 365 & $10.7 \times 10^{-3}$ & $6.1 \times 10^{9}$ & - & 17 \\
\hline & 650 & $25.5 \times 10^{-3}$ & $3.2 \times 10^{9}$ & & \\
\hline & 935 & 5.5 & $1.5 \times 10^{10}$ & & \\
\hline \multirow[t]{3}{*}{ Perovskite/ $/ \mathrm{TiO}_{2} / \mathrm{Si}$} & 350 & 0.07 & $5.5 \times 10^{12}$ & - & 18 \\
\hline & 800 & 0.87 & $6 \times 10^{12}$ & & \\
\hline & 1100 & 0.2 & $1.2 \times 10^{12}$ & & \\
\hline Perovskite & 680 & 230 & $1.7 \times 10^{10}$ & $4.2 / 9.4$ & This work \\
\hline Germanium & 1550 & 0.8 & $9.1 \times 10^{7}$ & $2.2 / 5.6$ & \\
\hline \multirow[t]{3}{*}{ Perovskite/Ge } & 405 & 395 & $2.8 \times 10^{10}$ & $4.1 / 9.2$ & \\
\hline & 680 & 228 & $1.6 \times 10^{10}$ & $1.8 / 5.1$ & \\
\hline & 980 & 32 & $2.2 \times 10^{9}$ & $2.1 / 5.7$ & \\
\hline
\end{tabular}

photodetector (PM100D, Thorlabs, Germany). The PL spectrum is characterized by confocal microscopy (LEICA DM $2700 \mathrm{M}$ ) and recorded by a spectrometer equipped with a CCD and a TCSPC detector (ANDORSR-500i-B1$\mathrm{R})$. The time-resolved $\mathrm{PL}$ spectrum measurements are performed by the TCSPC, in which a picosecond diode laser $(\lambda=405 \mathrm{~nm}, \approx 80 \mathrm{ps}, 20 \mathrm{MHz})$ is used as the excitation source and the overall time resolution is $\sim 250 \mathrm{ps}$. All measurements are performed at room temperature.

\section{Acknowledgements}

The authors are grateful to the National Natural Science Foundation of China (Nos. 61874109, 51525202, 61674140, 61574054, 51672076, 61804177) and the Foundation for Innovative Research Groups of NSFC (Grant 21521063). The authors thank Prof. Yu Huang (Hunan University) for the optical distribution simulation and Prof. Tian Jiang (NUDT) for the optical measurements.

\section{Author details}

${ }^{1}$ Key Laboratory for Micro-Nano Physics and Technology of Hunan Province, School of Physics and Electronics, Hunan University, Changsha, Hunan 410082, China. ${ }^{2}$ State Key Laboratory on Integrated Optoelectronics, Institute of Semiconductors, Chinese Academy of Sciences, Beijing 100083, China. ${ }^{3}$ Center of Materials Science and Optoelectronics Engineering, University of Chinese Academy of Sciences, Beijing 100049, China. ${ }^{4}$ Key Laboratory for Micro-Nano Physics and Technology of Hunan Province, College of Materials Science and Engineering, Hunan University, Changsha, Hunan 410082, China

\section{Author contributions}

W.H. and H.C. performed the fabrication of the device, experiments and measurements. W.H. and L.C. oversaw the growth of the perovskite material. Y.H. performed the optical simulation. W.H. and H.C. wrote the manuscript, with contributions from the other authors. A.P. and C.X. coordinated the project.

\section{Conflict of interest}

The authors declare that they have no conflict of interest.

Supplementary information is available for this paper at https://doi.org/ 10.1038/s41377-019-0218-y.

Received: 26 June 2019 Revised: 17 October 2019 Accepted: 4 November 2019

Published online: 21 November 2019

\section{References}

1. Sze, S. M. Coleman, D. J. Jr. \& Loya, A. Current transport in metalsemiconductor-metal (MSM) structures. Solid-State Electron. 14, 1209-1218 (1971).

2. Konstantatos, G. et al. Sensitive solution-processed visible-wavelength photodetectors. Nat. Photonics 1, 531-534 (2007).

3. Lin, C. H. \& Liu, C. W. Metal-insulator-semiconductor photodetectors. Sensors 10, 8797-8826 (2010).

4. Eda, G., Fanchini, G. \& Chhowalla, M. Large-area ultrathin films of reduced graphene oxide as a transparent and flexible electronic material. Nat. Nanotechnol. 3, 270-274 (2008). 
5. Park, S. et al. Flexible molecular-scale electronic devices. Nat. Nanotechnol. 7, 438-442 (2012).

6. De Arquer, F. P. G. et al. Solution-processed semiconductors for nextgeneration photodetectors. Nat. Rev. Mater. 2, 16100 (2017).

7. Assefa, S. et al. CMOS-integrated high-speed MSM germanium waveguide photodetector. Opt. Express 18, 4986-4999 (2010).

8. Chaisakul, P. et al. Integrated germanium optical interconnects on silicon substrates. Nat. Photonics 8, 482-488 (2014).

9. Chui, C. O., Okyay, A. K. \& Saraswat, K. C. Effective dark current suppression with asymmetric MSM photodetectors in Group IV semiconductors. IEEE Photonic Technol. Lett. 15, 1585-1587 (2003).

10. Okyay, A. K. et al. High-efficiency metal-semiconductor-metal photodetectors on heteroepitaxially grown Ge on Si. Opt. Lett. 31, 2565-2567 (2006).

11. Ang, K. W. et al. Novel silicon-carbon (Si:C) Schottky barrier enhancement layer for dark-current suppression in Ge-on-SOI MSM photodetectors. IEEE Electron Device Lett. 29, 704-707 (2008).

12. Yakimov, A. et al. Broadband Ge/SiGe quantum dot photodetector on pseudosubstrate. Nanoscale Res. Lett. 8, 217 (2013).

13. Qiao, $\mathrm{H}$. et al. Broadband photodetectors based on graphene- $\mathrm{Bi}_{2} \mathrm{Te}_{3}$ heterostructure. ACS Nano 9, 1886-1894 (2015).

14. Bai, F. et al. A high-performance self-powered photodetector based on monolayer $\mathrm{MoS}_{2}$ /perovskite heterostructures. Adv. Mater. Interfaces 5, 1701275 (2018).

15. Zheng, Z. et al. An enhanced UV-Vis-NIR an d flexible photodetector based on electrospun $\mathrm{ZnO}$ nanowire Array/PbS quantum dots film heterostructure. Adv. Sci. 4, 1600316 (2017)

16. Gao, T. et al. Performance-enhancing broadband and flexible photodetectors based on perovskite/ZnO-nanowire hybrid structures. Adv. Optical Mater. 5, 1700206 (2017).

17. Chen, S. et al. A flexible UV-Vis-NIR photodetector based on a perovskite/ conjugated-polymer composite. Adv. Mater. 28, 5969-5974 (2016).

18. Cao, F. R. et al. Novel perovskite/ $/ \mathrm{TiO}_{2} / \mathrm{Si}$ trilayer heterojunctions for highperformance self-powered ultraviolet-visible-near infrared (UV-Vis-Nir) photodetectors. Nano Res. 11, 1722-1730 (2018).

19. Lee, Y. et al. High-performance perovskite-graphene hybrid photodetector. Adv. Mater. 27, 41-46 (2015).

20. Yang, F. et al. Ultrathin broadband germanium-graphene hybrid photodetector with high performance. ACS Appl. Mater. Interfaces 9, 13422-13429 (2017).

21. Song, X. F. et al. Boosting two-dimensional $\mathrm{MoS}_{2} / \mathrm{CsPbBr} 3$ photodetectors via enhanced light absorbance and interfacial carrier separation. ACS Appl. Mater. Interfaces 10, 2801-2809 (2018)

22. Wei, Y. Z. et al. Hybrid organic/PbS quantum dot bilayer photodetector with low dark current and high detectivity. Adv. Funct. Mater. 28, 1706690 (2018).

23. Alwadai, N. et al. High-performance ultraviolet-to-infrared broadband perovskite photodetectors achieved via inter-/intraband transitions. ACS Appl. Mater. Interfaces 9, 37832-37838 (2017).

24. De Wolf, S. et al. Organometallic halide perovskites: sharp optical absorption edge and its relation to photovoltaic performance. J. Phys. Chem. Lett. 5, 1035-1039 (2014).

25. Stranks, S. D. et al. Electron-hole diffusion lengths exceeding 1 micrometer in an organometal trihalide perovskite absorber. Science 342, 341-344 (2014).

26. Li, N. X. et al. Cation and anion immobilization through chemical bonding enhancement with fluorides for stable halide perovskite solar cells. Nat. Energy 4, 408-415 (2019)

27. Zhou, Y. et al. Ultra-broadband optical amplification at telecommunication wavelengths achieved by bismuth-activated lead iodide perovskites. J. Mater. Chem. C. 5, 2591-2596 (2017).

28. Xie, $Y$. et al. Giant two-photon absorption in mixed halide perovskite $\mathrm{CH}_{3} \mathrm{NH}_{3} \mathrm{~Pb}_{0.75} \mathrm{Sn}_{0.25} \mathrm{l}_{3}$ thin films and application to photodetection at optical communication wavelengths. Adv. Optical Mater. 6, 1700819 (2018).
29. Song, J. Z. et al. Organic-Inorganic hybrid passivation enables perovskite QLEDs with an EQE of 16.48\%. Adv. Mater. 30, 1805409 (2018).

30. Jiang, Q. et al. Surface passivation of perovskite film for efficient solar cells. Nat Photonics 13, 460-466 (2019).

31. Wang, Y. B. et al. Stabilizing heterostructures of soft perovskite semiconductors. Science 365, 687--691 (2019).

32. Best Research-Cell Efficiency Chart. https:/www.nrel.gov/pv/cell-efficiency. html.

33. Dou, L. T. et al. Solution-processed hybrid perovskite photodetectors with high detectivity. Nat. Commun. 5, 5404 (2014).

34. Dong, R. et al. High-gain and low-driving-voltage photodetectors based on organolead triiodide perovskites. Adv. Mater. 27, 1912-1918 (2015).

35. Xia, H. R. et al. Organohalide lead perovskite based photodetectors with much enhanced performance. Chem. Commun. 50, 13695-13697 (2014).

36. Saidaminov, M. I. et al. Perovskite photodetectors operating in both narrowband and broadband regimes. Adv. Mater. 28, 8144-8149 (2016).

37. $\mathrm{Hu}, \mathrm{W}$. et al. Solvent-induced crystallization for hybrid perovskite thin-film photodetector with high-performance and low working voltage. J. Phys. D: Appl. Phys. 50, 375101 (2017)

38. Wang, W. H., Ma, Y. R. \& Qi, L. M. High-performance photodetectors based on organometal halide perovskite nanonets. Adv. Funct. Mater. 27, 1603653 (2017).

39. Zhou, J. C. \& Huang, J. Photodetectors based on organic-inorganic hybrid lead halide perovskites. Adv. Sci. 5, 1700256 (2018).

40. Lin, Y. H. et al. Metal-halide perovskite transistors for printed electronics: challenges and opportunities. Adv. Mater. 29, 1702838 (2017).

41. Fang, Y. J. et al. Highly narrowband perovskite single-crystal photodetectors enabled by surface-charge recombination. Nat. Photonics $\mathbf{9}$, 679-686 (2015).

42. Sutherland, B. R. et al. Sensitive, fast, and stable perovskite photodetectors exploiting interface engineering. ACS Photonics 2, 1117-1123 (2015).

43. Hu, W. et al. High-performance flexible photodetectors based on high-quality perovskite thin films by a vapor-solution method. Adv. Mater. 29, 1703256 (2017).

44. Cong, H. et al. Silicon based GeSn p-i-n photodetector for SWIR detection. IEEE Photonics J. 8, 6804706 (2016).

45. $\mathrm{Yu}, \mathrm{K}$. et al. Fabrication of high-hole-mobility germanium-on-insulator wafers through an easy method. J. Alloy. Compd. 750, 182-188 (2018).

46. Walheim, S. et al. Nanophase-separated polymer films as high-performance antireflection coatings. Science 283, 520-522 (1999).

47. Choi, $K$. et al. Nano-tailoring the surface structure for the monolithic high-performance antireflection polymer film. Adv. Mater. 22, 3713-3718 (2010).

48. Yang, J. et al. Design and fabrication of broadband ultralow reflectivity black Si surfaces by laser micro/nanoprocessing. Light. Sci. Appl. 3, e185 (2014).

49. Onoda-Yamamuro, N., Matsuo, T. \& Suga, $\mathrm{H}$. Dielectric study of $\mathrm{CH}_{3} \mathrm{NH}_{3} \mathrm{PbX}_{3}(X$ $=\mathrm{Cl}, \mathrm{Br}$, I). J. Phys. Chem. Solids 53, 935-939 (1992).

50. Yang, A. L. et al. Investigation of optical and dielectric constants of organicinorganic $\mathrm{CH}_{3} \mathrm{NH}_{3} \mathrm{Pbl}_{3}$ perovskite thin films. J. Nanomed. Nanotechnol. 7, 1000407 (2016).

51. Macleod, H. A. Thin-Film Optical Filters. (Adam Hilger, Bristol, UK, 1986).

52. Butler, K. T., Frost, J. M. \& Walsh, A. Band alignment of the hybrid halide perovskites $\mathrm{CH}_{3} \mathrm{NH}_{3} \mathrm{PbCl}_{3}, \mathrm{CH}_{3} \mathrm{NH}_{3} \mathrm{PbBr}_{3}$ and $\mathrm{CH}_{3} \mathrm{NH}_{3} \mathrm{Pbl}_{3}$. Mater. Horiz. 2, 228-231 (2015)

53. Yamaha, T. et al. Experimental observation of type-l energy band alignment in lattice-matched $\mathrm{Ge}_{1-x-y} \mathrm{Si}_{x} \mathrm{Sn}$ / $/ \mathrm{Ge}$ heterostructures. Appl. Phys. Lett. 108, 061909 (2016).

54. Kim, J., Lee, J. H. \& Hong, K. H. A pathway to type-l band alignment in Ge/Si core-shell nanowires. J. Phys. Chem. Lett. 4, 121-126 (2013).

55. Buyanova, I. A. et al. Type I band alignment in the $\mathrm{GaN}_{x} \mathrm{As}_{1-x} / \mathrm{GaAs}$ quantum wells. Phys. Rev. B 63, 033303 (2001). 